\title{
Depresión en el personal de custodia y vigilancia de un establecimiento penitenciario de mediana seguridad y carcelario, 2019
}

\author{
Ángely Alejandra Ángel Delgado'; Mateo Arango Londoño²; \\ Tutora: Daris Osuna Julio ${ }^{3}$
}

RESUMEN La depresión es un trastorno mental que constituye un problema de salud pública y afecta a millones de personas en todo el mundo; se asocia a problemas y conductas no deseables difíciles de eliminar, contribuyendo a una carga mundial de discapacidad y mortalidad. Para describir la depresión en el personal de custodia y vigilancia de un establecimiento penitenciario de mediana seguridad y carcelario 2019, se realizará un estudio descriptivo con enfoque cuantitativo, aplicando una encuesta basada en el cuestionario Hamilton para la depresión (HAM-D); la información se procesará en el paquete estadístico SPSS. Entre los resultados esperados está la identificación de la depresión en dicha población, el grado de depresión de la población estudio, y se establecerán los síntomas de la depresión en dicha población.

Cárcel;

PALABRAS Depresión;

CLAVE Personal de Custodia;

Vigilancia

1 Estudiante. Fundación Universitaria del Área Andina Seccional Pereira.

2 Estudiante Terapia Respiratoria IV Semestre. Fundación Universitaria del Área Andina Seccional Pereira. 


\section{Depression in the custody and surveillance personnel of a medium security and prison facility, 2019}

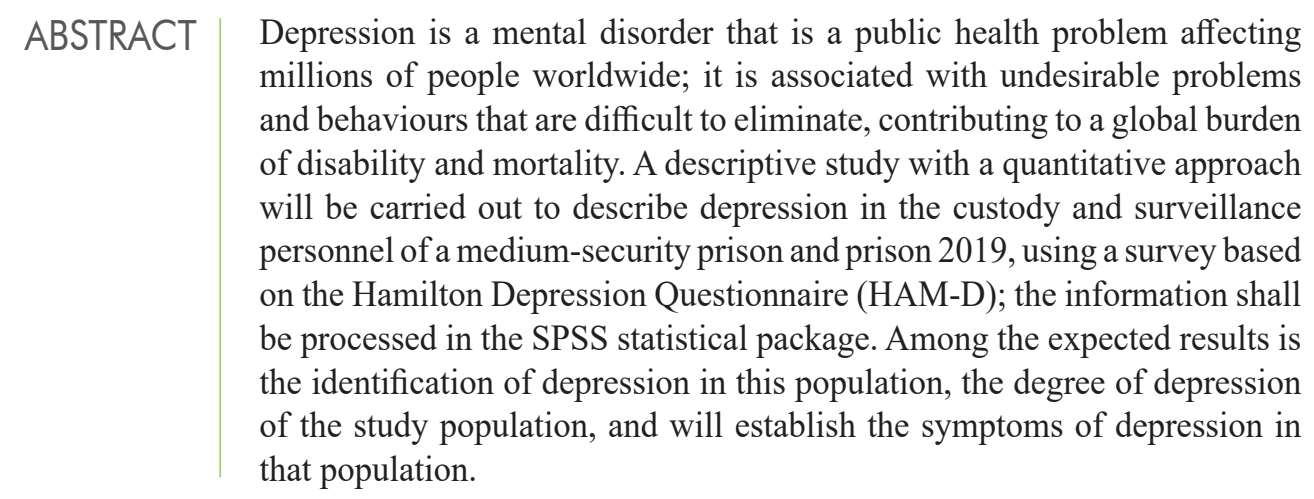

Prison;

Depression;

Personnel of the Custody;

Vigilance 


\section{INTRODUCCIÓN}

Según la Organización Mundial de la Salud (OMS), la depresión es una enfermedad frecuente en todo el mundo, y se calcula que afecta a más de 300 millones de personas. Establece que la depresión es distinta a las variaciones habituales del estado de ánimo y de las respuestas emocionales breves a los problemas de la vida cotidiana. La depresión es un problema de salud serio, especialmente si es de larga duración e intensidad, porque puede causar gran sufrimiento y alterar las actividades laborales, escolares y familiares, y en el peor de los casos puede llevar al suicidio. Se estima que cada año se suicidan alrededor de 800.000 personas en el mundo, y se ha convertido en la segunda causa de muerte en personas de 15 a 29 años de edad (1).

El informe de salud mental en la región de las Américas, registra que el suicidio es una posible consecuencia importante de los trastornos mentales, y se estima que la tasa de mortalidad en la región es de 7,3 por 100.000 habitantes, más de $90 \%$ de las víctimas de suicidio padecen un trastorno mental crónico, susceptible de ser diagnosticado como la depresión, y otros trastornos derivados del uso de sustancias (2).

En el informe sobre la carga de trastornos mentales en la región de las Américas 2018, establece que la principal causa de los años de vida ajustados en función a la discapacidad (AVAD) por trastornos mentales, son los trastornos depresivos, que representan 3,4\% del total de AVAD, seguidos por los trastornos de ansiedad, con el $2,1 \%$, por lo tanto, se considera que los episodios depresivos graves son el quinto estado de salud humana más discapacitante. En Colombia el estudio de estimación de la carga de enfermedad realizado en el 2010, encontró que en las personas de 15 a 29 años, la depresión mayor unipolar fue la primera causa de carga de la enfermedad con 168 años de vida saludable perdidos, probablemente los centros penitenciarios del país no cuenten con espacios para que los dragoneantes ejerzan su trabajo en un ambiente psicosocial adecuado, ya que los esfuerzos y gran parte de investigaciones realizadas están centradas en la población privada de la libertad, y quizás las estrictas normas de cumplimiento laboral, jornadas extensas de trabajo y el encierro, pueden influir de manera negativa en la salud mental de los trabajadores.

Por lo tanto, es relevante hacer un estudio detallado sobre el desarrollo de esta problemática en el personal de custodia y vigilancia del establecimiento penitenciario, el cual pretende encaminar acciones hacia la promoción y prevención de factores que conllevan al desarrollo de la depresión, ya que el objetivo de salud pública es impulsar una cultura de autocuidado y de corresponsabilidad social en torno a la salud mental, y de esta forma reducir los AVISAS por trastornos mentales y del comportamiento en población general de 15 a 65 años, a 55 por 1.000 personas, establecido en el Plan Decenal de Salud Pública en sus dimensiones prioritarias en la protección de la salud en los trabajadores, convivencia social y salud mental (5).

Los trastornos depresivos pueden clasificarse en dos subcategorías: en primer lugar, como trastorno o episodio depresivo mayor, que presenta síntomas como estado de ánimo deprimido, pérdida de interés y de la capacidad de disfrutar, disminución de la energía; en segundo lugar, distimia, forma persistente o crónica de depresión leve, 142 donde los síntomas tienden a ser menos intensos y más duraderos (6). 
Según Piqueras et al, quien a su vez cita a Beck et al, Ellis, afirman que la forma clínica de la depresión es manifestada como un trastorno del estado de ánimo, caracterizado por una sensación de tristeza intensa superior a dos meses, que se produce por diversas causas, como acontecimientos de la vida diaria, cambios químicos en el cerebro, efectos secundarios a medicamentos y diversos trastornos físicos o médicos (7). Por lo cual afirma Piqueras, que la depresión es el resultado final de la interacción de múltiples constitucionales, evolutivos, ambientales e interpersonales, que modifican las pautas de neurotransmisores entre los hemisferios cerebrales y el sistema límbico y que conductualmente la depresión se caracteriza principalmente por la reducción generalizada en la frecuencia de la conducta, que a su vez sería la consecuencia de una pérdida de reforzadores positivos o de la incapacidad de hacer frente a una situación, y que desde el punto de vista cognitivo la idea fundamental es que la depresión depende de cómo el individuo interpreta los acontecimientos que le ocurren (7).

Tal y como lo indica Puerta et al, "que la depresión es por excelencia el trastorno mental más común presente en el mundo actual, si bien existe variación respecto al grado de debilitamiento que manifiestan quienes padecen un episodio depresivo mayor, debe haber ya sea una angustia significativa desde el punto de vista clínico o algún decremento en áreas sociales, familiares u otros de funcionamientos cotidiano para declarar el diagnóstico y que en casos extremos la persona no es capaz de procurar el mínimo cuidado o higiene personal (8)".

Según el Manual Diagnóstico y Estadístico de los Trastornos Mentales -5 (DSM5), los trastornos depresivos en adultos se dividen en trastorno depresivo mayor, persistente (distimia), trastorno disfórico premenstrual, trastorno depresivo inducido por sustancias o medicamentos u otra condición médica, otros trastornos depresivos especificados y no especificados.

La característica del trastorno depresivo mayor, es la presencia en la persona durante un periodo de al menos dos semanas consecutivas, de un estado de ánimo triste, deprimido o una pérdida acusada del interés o del placer en todas en todas o casi todas las actividades con las que antes solía disfrutar. Además, el trastorno depresivo persistente se caracteriza por un estado de ánimo depresivo persistente, la mayor parte del día, casi todos los días, aunque de menor gravedad que el trastorno depresivo mayor, que es manifestado por la persona u observado por los demás durante al menos dos años (9).

Igualmente el DSM-5 agrupa en cinco categorías distintas la sintomatología de la depresión, según las manifestaciones generadas en cada persona de la siguiente manera: síntoma afectivo y emocional que se puede manifestar como desesperanza, pesimismo, abatimiento emocional y amargura; síntomas cognitivos que se pueden presentar como problemas de atención, concentración y memoria; síntomas motivacionales reflejados en la persona como aburrimiento, indiferencia, apatía, incapacidad para disfrutar de las cosas o experimentar placer, y por último, los síntomas somáticos tales como dolor de cabeza y musculares, visión borrosa, sequedad de boca y molestias gastrointestinales.

De igual modo la Asociación Americana de Psiquiatría, define los trastornos mentales como un síndrome caracterizado por una alteración clínicamente significativa del 
estado cognitivo, la regulación emocional o el comportamiento de un individuo, que refleja una disfunción de los procesos psicológicos, biológicos o del desarrollo que subyacen en su función mental, además los trastornos mentales van asociados a un estrés significativo o una discapacidad, ya sea social, laboral o de otras actividades importantes (10). Así mismo el Ministerio de Salud y Protección Social en la Ley 1616, define la Salud Mental como "un estado dinámico que se expresa en la vida cotidiana a través del comportamiento y la interacción de manera tal que permite a los sujetos individuales y colectivos desplegar sus recursos emocionales, cognitivos y mentales para transitar por la vida cotidiana, para trabajar, establecer relaciones cognitivas y para contribuir a la comunidad" (11).

\section{METODOLOGÍA}

La presente investigación tendrá un enfoque cuantitativo y un diseño descriptivo en el establecimiento penitenciario de mediana seguridad y carcelario; el tamaño de muestra será de 110 dragoneantes y se establecerá con un nivel de confianza del $5 \%$ y un error del 4\%. Previa firma del consentimiento informado se aplicará un instrumento basado en el cuestionario Hamilton para la depresión (HAM-D); la información se procesará en el paquete estadístico SPSS.

Para dar cumplimiento a la reglamentación sobre aspectos, se revisará el informe Belmont como la declaración de Singapur, Helsinki y la Resolución 008430 de 1993.

De acuerdo a la norma ISO 26000 de 2012, el equipo investigador asumió como responsabilidad social el acompañamiento a la institución carcelaria de actividades dirigidas a intervenir la aparición de síntomas depresivos, por medio de una jornada de sensibilización.

\section{RESULTADOS ESPERADOS}

Identificar la presencia de depresión en el personal de custodia y vigilancia; obtener el grado de depresión de la población estudio y establecer los síntomas clínicos de la depresión en dicha población

\section{IMPACTOS}

Entre los impactos esperados está: detectar a tiempo la presencia de depresión en los funcionarios de custodia y vigilancia y para realizar una propuesta de intervención. Capacitar a la población con depresión sobre la cultura de autocuidado y salud mental; crear cultura de corresponsabilidad social en torno a la salud mental en los servidores penitenciarios para generar procesos de apropiación y formación sobre los factores desencadenantes de depresión. 
1. (OMS) Depresión [internet]. Available from: https://www.who.int/es/newsroom/fact-sheets/detail/depression

2. Estado de salud de la población, la salud mental en la región de las Américas 2013. [internet]. Available from: https://www.paho.org/salud-en-las-americas2017/?post t es=la-salud-mental-en-la-region-de-las-americas\&lang=es

3. La carga de los trastornos mentales en la región de las Américas 2018. [internet]. 2018. 14 y 15 p. Available from: http://iris.paho.org/xmlui/bitstream/ handle $/ 123456789 / 49578 / 9789275320280$ spa.pdf? sequence $=9 \&$ isAllowed $=y$

4. Boletín de salud mental-depresión-subdirección de enfermedades no trasmisibles 2017. [internet]. Available from: https://www.minsalud.gov.co/sites/rid/Lists/ BibliotecaDigital/RIDE/VS/PP/ENT/boletin-depresion-marzo-2017.pdf

5. Plan Decenal de Salud Pública 2012-2021. [internet]. Available from: https://www.minsalud.gov.co/Documentos $\% 20 \mathrm{y} \% 20$ Publicaciones/ Plan\%20Decenal\%20-\%20Documento\%20en\%20consulta\%20para\%20 aprobaci\%C $3 \% \mathrm{~B} 3 n . p d f$

6. (OMS). Depresión y otros trastornos mentales comunes. Estimaciones sanitarias mundiales. 2017. [internet]. Available from: http://iris.paho. org/xmlui/bitstream/handle/123456789/34006/PAHONMH17005-spa. pdf? sequence $=1$ \&isAllowed $=\mathrm{y}$

7. Piqueras et al. ansiedad, depresión y salud. Suma psicológica. [internet]. 2008. [Consultado 6 abril 2019]. Available from: https://www.redalyc.org/articulo. oa? id $=134212604002$

8. Puerta, J; Padilla, D. Terapia cognitiva-conductual (TCC) como tratamiento para la depresión. DUAZARY. [internet]. 2011. [Consultado 7 abril 2019]. Available from: https://www.redalyc.org/articulo.oa?id=512156315016

9. Ortiz-Tallo M, editor. Psicopatología clínica: adaptado al DSM-5. Madrid: Difusora Larousse - Ediciones Pirámide; 2013.

10. Asociación americana de psiquiatría. Manual diagnóstico y estadístico de los trastornos mentales. Medica panamericana. 2013. [Consultado 5 abril 2019].

11. Ministerio de salud y protección social. Ley 161621 enero 2013. [internet]. [Consultado 6 abril 2019]. Available from: https://www.minsalud.gov.co/sites/ $\underline{\mathrm{rid} / \text { Lists/BibliotecaDigital/RIDE/DE/DIJ/ley-1616-del-21-de-enero-2013.pdf }}$ 